\title{
Evaluación del triflumurón y la mezcla de Bacillus thuringiensis más Bacillus sphaericus para el control de las formas inmaduras de Aedes aegypti y Culex quinquefasciatus en sumideros en Cali, Colombia
}

\author{
Gloria I. Giraldo-Calderón ${ }^{1,2}$, Mauricio Pérez¹, Carlos A. Morales³, Clara B. Ocampo ${ }^{1}$ \\ 1 Unidad de Entomología Médica, Centro Internacional de Entrenamiento e Investigaciones Médicas (CIDEIM), \\ Cali, Colombia \\ 2 Department of Entomology, Purdue University, West Lafayette, Indiana, United States of America \\ 3 Secretaría de Salud Pública Municipal de Cali, Cali, Colombia
}

Introducción. En Cali los sumideros son uno de los principales criaderos de Aedes aegypti y Culex quinquefasciatus que son controlados por la Secretaría de Salud Municipal utilizando el insecticida triflumurón desde 1999. Se sospecha falla al tratamiento.

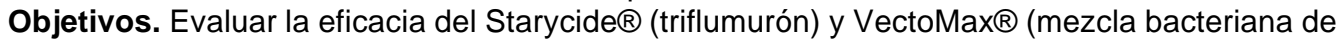
Bacillus thuringiensis var. israelensis y Bacillus sphaericus) en el control de $A$. aegypti y $C$. quinquefasciatus en los sumideros y determinar el efecto residual de una única aplicación de VectoMax®, en épocas de alta y baja pluviosidad.

Materiales y métodos. La eficacia de los productos fue medida en 60 sumideros de una zona residencial de Cali por un período de 90 días. La media de individuos inmaduros (larvas y pupas de $A$. aegypti y $C$. quinquefasciatus) fueron obtenidas quincenalmente de 40 sumideros intervenidos (20 con triflumurón y 20 con VectoMax®) y 20 sin tratamiento (grupo testigo). EI efecto residual de la mezcla bacteriana se evaluó quincenalmente en 10 sumideros en cada temporada climática.

Resultados. Los sumideros tratados con VectoMax $\AA^{\circledR}$ presentaron diferencias en el promedio de estadios inmaduros en ambas especies frente al testigo $(p<0,01)$. En contraste, el tratamiento con triflumurón sólo presentó diferencias en los estadios inmaduros de $A$. aegypti con respecto al testigo $(p<0,001)$. El efecto residual del VectoMax $\Theta$ fue mayor en la época de baja pluviosidad con respecto al testigo $(p<0,001)$.

Conclusión. La mezcla bacteriana fue el tratamiento más eficaz en el control de ambas especies durante el período evaluado (15 días).

Palabras clave: control biológico de plagas, eficacia, Bacillus, Aedes, Culex.

Evaluation of the triflumuron and the mixture of Bacillus thuringiensis plus Bacillus sphaericus for control of the immature stages of Aedes aegypti and Culex quinquefasciatus (Diptera: Culicidae) in catch basins

Introduction. In Cali, Colombia, catch basins (streetside storm drains) are one of the main larval habitats of Aedes aegypti and Culex quinquefasciatus. Since 1999, these mosquitoes have been controlled by the Secretaría de Salud Municipal (Secretary of Municipal Public Health) using the larvicide triflumuron. Because of high densities of these mosquitoes that remain in the city, treatment failure was suspected -possibly insecticide resistance of the target species. Objectives. The efficacy of triflumuron and VectoMax® (biorational mixture of Bacillus thuringiensis var. israelensis plus Bacillus sphaericus) were evaluated in the control of $A$. aegypti and $C$. quinquefasciatus in catch basins. The residual effect of a single application of the biorational formulation was determined in catch basins during periods of high and low rainfall.

Materials and methods. The efficacy of the products was measured in 60 catch basins located in a residential neighborhood of Cali for a period of 90 days. The mean number of immature 
instars ( $A$. aegypti and $C$. quinquefasciatus larvae and pupae of both species) was determined biweekly from 40 catch basins with insecticide intervention (20 treated with triflumuron, 20 with VectoMax $\AA$ ) and 20 untreated (control group). The residual effect of the biorational larvicide was evaluated biweekly in 10 catch basins during each of the 2 climatic periods.

Results. The catch basins treated with VectoMax® presented a significantly lower mean number of immature instars of both species compared with the control $(p<0.01)$. In contrast, the triflumuron treatment significantly reduced only immature instars of $A$. aegypti compared with the control $(p<0.001)$. The residual effect of VectoMax $®$ was higher during low rainfall compared to the control $(p<0.001)$.

Conclusion. The biorational formulation was the more effective treatment for the control of both species during the period of evaluation (15 days).

Key words: pest control, biological, efficacy, Bacillus, Aedes, Culex.

El control de los mosquitos vectores es una estrategia para la reducción de las enfermedades transmitidas por estos agentes (1). Mundialmente, la herramienta principal para el control de insectos adultos e inmaduros es el uso de insecticidas químicos, pero el aumento de la resistencia hace que cada día sean menos efectivos (2-4). Entre los retos que presenta este fenómeno está la necesidad de utilizar nuevos productos, los cuales son cada día más escasos, pueden presentar un alto costo y, en algunos casos, aumento de la toxicidad o efectos adversos en organismos no blanco $(2,4)$. Todo lo anterior dificulta el manejo de los programas de control y abre la necesidad de entender mejor la biología de los vectores y buscar estrategias para el manejo integrado de plagas (integrated pest management) (3). Además, es necesaria la evaluación continua de la efectividad de los insecticidas de uso común en los programas de control y la búsqueda de insecticidas alternos.

Aedes aegypti y Culex quinquefasciatus son las especies de mosquitos de importancia en salud pública en Cali, Colombia. A. aegypti es el responsable de la transmisión del virus del dengue en la ciudad y $C$. quinquefasciatus es una peste debido a su comportamiento antropofílico nocturno, que presenta un riesgo potencial para la transmisión de enfermedades emergentes como el virus del Nilo occidental $(5,6)$. Uno de los principales

Correspondencia:

Clara Beatriz Ocampo, Unidad de Entomología Médica, Centro Internacional de Entrenamiento e Investigaciones Médicas CIDEIM, Avenida 1 Norte № 3-03, Cali, Colombia. Teléfono: 668 2164; telefax: 6672989

claraocampo@cideim.org.co

Recibido: 30/04/07; aceptado:05/12/07 sitios de cría de estos mosquitos en Cali son los sumideros colectores de aguas lluvias, los cuales han sido identificados como los mayores productores de pupas de ambas especies en comparación con los criaderos intradomiciliares (González R, Suárez MF. Sewers: The principal Aedes aegypti breeding sites in Cali, Colombia. American Society of Tropical Medicine and Hygiene (ASTMH) Annual Meeting 44th. Texas, USA, November 17-21, 1995:217). Con este estudio los sumideros se identificaron como una amenaza permanente para la salud pública de Cali y, por esta razón, en 1993 se inició un programa de control de vectores, el cual, desde 1998 ha estado enfocado en la aplicación quincenal de larvicidas en los 49.000, aproximadamente, sumideros de la ciudad (comunicación personal, Secretaría de Salud Pública Municipal de Cali).

Los sumideros son trampas de sólidos del sistema colector de aguas lluvias distribuidos en toda la ciudad para evitar la obstrucción del alcantarillado. Presentan en promedio una capacidad máxima para almacenar 250 litros de agua $(1 \mathrm{~m} \times 0,5 \mathrm{~m} \times$ $0,5 \mathrm{~m}$ ) y un diseño que permite las condiciones para el desarrollo de los estadios inmaduros de los mosquitos durante todo el año (7). El principal producto utilizado desde 1999 para el control de los sumideros ha sido el triflumurón. Este insecticida es un regulador del desarrollo de los insectos (insect growth regulator), cuyo mecanismo de acción es interferir en la síntesis de la quitina, polisacárido estructural en la formación del exoesqueleto de los artrópodos $(8,9)$.

Este producto ha demostrado buenos resultados para el control de mosquitos en otros estudios, como, por ejemplo, en Burkina Faso, el triflumurón 
granulado (1,17\% ingrediente activo) inhibió completamente la emergencia de adultos de $C$. quinquefasciatus por 30 días (10) y en India, la aplicación de triflumurón en los hábitat de cría naturales en aguas limpias y contaminadas a una dosis de 0,5 ppm produjo una inhibición completa de la emergencia de adultos de Anopheles spp. y Culex spp. entre tres y siete semanas (9).

En Cali, en el momento del estudio, se sospechaba falla al tratamiento en $C$. quinquefasciatus, debido a la permanencia de sumideros positivos para esta especie a pesar del tratamiento continuo (comunicación personal, C. A. Morales). Esto hizo necesario evaluar la eficacia del tratamiento actual e identificar nuevas estrategias de control.

El presente estudio evaluó la eficacia de la intervención actual con triflumurón contra un nuevo tratamiento, el VectoMax® que consiste en una mezcla bacteriana compuesta de Bacillus thuringiensis var. israelinsis y Bacillus sphaericus cepa 2362 en una presentación de $10 \mathrm{~g}$ (producto de Valent-Bioscience en experimentación en el momento del estudio, el cual fue registrado en la Environmental Protection Agency como VectoMax® VBC60035 en el 2006). EI VBC60035 busca controlar las larvas de $A$. aegypticon $B$. thuringiensis var. israelinsis y las larvas de $C$. quinquefasciatus con $B$. sphaericus (11). La ingestión de este biolarvicida causa la muerte de las larvas por inanición en 24 o 48 horas debido a que los cristales tóxicos producidos por las bacterias perforan el intestino (11).

Además de la evaluación de la eficacia, se evaluó el efecto residual de la mezcla bacteriana a través de una única aplicación en los sumideros durante una época de alta y otra de baja pluviosidad. La evaluación de los sumideros testigo (libres de tratamiento), además de evaluar la eficacia de los tratamientos, permitió observar la dinámica de las densidades de población relativas de larvas y pupas de $A$. aegypti y $C$. quinquefasciatus bajo diferentes condiciones climáticas.

\section{Materiales y métodos}

\section{Área de estudio}

El estudio se llevó a cabo en el municipio de Santiago de Cali, capital del departamento del
Valle del Cauca, una de las ciudades más pobladas del país, con 2'068.386 habitantes. La ciudad está ubicada a una altura de $995 \mathrm{~m}$, presenta una temperatura media anual de $23^{\circ} \mathrm{C}$ y una precipitación promedio anual de $1.153 \mathrm{~mm}$, con dos picos de lluvias usualmente entre marzomayo y octubre-diciembre (12). La ciudad está clasificada como uno de los focos hiperendémicos para dengue en Colombia debido a la circulación de los cuatro serotipos y su incidencia anual, que para 2005 fue de 90,3 casos por 100.000 habitantes (13-15).

\section{Diseño del estudio}

Se evaluó la eficacia del VBC60035 y el tratamiento actual, el triflumurón, con respecto a un grupo testigo (sin tratamiento). Para esto se seleccionó un barrio residencial de la ciudad en donde no se habían registrado casos de dengue en los últimos dos años. Durante el estudio se suspendieron las actividades de control en los sumideros ejercidas por la Secretaría de Salud Pública Municipal de Cali.

El barrio se dividió en cuatro zonas de 20 sumideros cada una, para evaluar las intervenciones (triflumurón, VBC60035, efecto residual del VBC60035) contra una zona testigo. Las zonas de intervención estaban separadas por, al menos, dos calles ( $200 \mathrm{~m}$ ) cuyos sumideros no fueron incluidos en el estudio. Las zonas asignadas para las intervenciones se seleccionaron de forma aleatoria.

Las intervenciones se realizaron durante los meses de mayo a agosto de 2005, periodo que cubrió una época de lluvia (mayo-junio) y una época seca (julio-agosto) con promedios de precipitación diaria de $3,96 \mathrm{~mm}$ (rango $39,3-1,5 \mathrm{~mm}$ ) y $0,73 \mathrm{~mm}$ (rango $11-1,2 \mathrm{~mm}$ ), respectivamente (16).

Las intervenciones con triflumurón y VBC60035 se aplicaron en 20 sumideros cada una y se evaluaron quincenalmente durante todo el período del estudio. El efecto residual de la mezcla bacteriana se evaluó en 10 sumideros después de una única aplicación en la época de alta pluviosidad (26 de mayo) y en otros 10 en la época de baja pluviosidad (7 de julio), por medio de revisiones quincenales, para conocer la tasa de 
recolonización de $A$. aegyptiy $C$. quinquefasciatus en cada caso.

El efecto del tratamiento de la mezcla bacteriana en todas las intervenciones se evaluó 72 horas después de la aplicación. Los sumideros testigo se evaluaron quincenalmente para identificar la dinámica y la relación de $A$. aegypti y $C$. quinquefasciatus en estos criaderos durante todo el estudio. Las evaluaciones se hicieron quincenalmente debido a que es el período mínimo en el cual la Secretaría de Salud Pública Municipal de Cali puede mantener el programa de control.

\section{Recolección de muestras y manejo en el laboratorio}

Muestreo: para el muestreo de los sumideros se adaptó una metodología a partir de experiencias previas compartidas por Peter DeChant y Ranulfo González (comunicación personal). Las muestras en los sumideros se obtuvieron con una red de acuario con dimensiones de $8 \mathrm{~cm} \times 10 \mathrm{~cm}$, unida a un cabo largo $(\sim 1,5 \mathrm{~m})$ para facilitar su manipulación. El uso de esta red permitió no destapar el sumidero ya que cabe entre sus rejillas. Rápidamente la red se sumergía $10 \mathrm{~cm}$, aproximadamente, bajo el agua. La red se desplazaba haciendo un movimiento en forma de ocho, a excepción de los sumideros que estaban llenos de elementos flotantes (desechos y materia orgánica), que no permitían hacer esto, por lo cual el movimiento se hacia en forma lineal. Posteriormente, la red se retiraba del sumidero para lavarla sobre un recipiente y almacenar las muestras para el conteo de larvas y pupas de $A$. aegypti y $C$. quinquefasciatus en el laboratorio. Se esperaban cinco minutos entre cada toma de muestra, con la precaución de alejarse para no hacer sombra sobre el sumidero y de esta forma conseguir que las larvas y pupas volvieran a la superficie.

Tamaño de la muestra: por capacidad logística, se evaluaron 20 sumideros por tratamiento por un periodo de 90 días. Se obtuvieron cuatro muestras de agua de cada sumidero en cada observación. Esta cifra se determinó después de hacer un muestreo piloto en 36 sumideros, en los cuales se tomaron 10 muestras por cada uno. Para cada una de estas 10 muestras, se contó el número de larvas de tercero y cuarto estadio y pupas (sin distinguir especie).

Utilizando los porcentajes acumulados durante la primera hasta la última muestra (no se presentan los datos) se determinó que al tomar las primeras cuatro muestras por cada sumidero, se recolectaba, aproximadamente, el $80,7 \%$ de larvas y $88,5 \%$ de las pupas de ambas especies. A partir de este momento las muestras se tomaron en cada una de las esquinas del sumidero, teniendo en cuenta que Sih (17) describe que $A$. aegypti y C. pipiens evitan el centro de un recipiente. Esta estandarización concuerda con la realizada en estudios previos en esta misma ciudad por SuárezRubio y M. Suárez (7) y Satizábal Rengifo (18); sin embargo, estos dos autores no emplearon red sino un cucharón de $350 \mathrm{ml}$ o $500 \mathrm{ml}$.

Insecticidas: la dosis empleada para el triflumurón (Starycide ${ }^{\circledR} 480 \mathrm{SC}$ ) fue de $5 \mathrm{ml}$ para una concentración final de $0,048 \mathrm{mg}$ de ingrediente activo por litro (ppm) actualmente empleada por la Secretaría de Salud Pública Municipal de Cali (19). EL VBC60035 se aplicó en la dosis de $10 \mathrm{~g}$ en bolsas hidrosolubles suministrada por Valent BioScience Corporation.

Manejo de los datos: para cada sumidero se contó el total de las larvas de tercero y cuarto estadio de $A$. aegypti, $C$. quinquefasciatus y pupas $\sin$ distinguir especie. Las pupas recolectadas se mantuvieron en el laboratorio hasta su emergencia, para identificar los adultos y evaluar el insecto. A cada sumidero estudiado se le registró la profundidad del agua y la presencia o ausencia de aceite. Las especies de larvas y adultos se identificaron mediante claves taxonómicas de los Centers for Disease Control and Prevention (CDC) (20) y Lane (21).

\section{Análisis estadístico}

Los datos de campo y de laboratorio se registraron en una base de datos en Access (Microsoft ${ }^{\circledR}$ Office Acces 2003) y, posteriormente, se analizaron en SPSS 7.5 para Windows (SPSS Inc., Chicago, 1996) y STATA 6.0 (STATA Corporation, 1999).

Para la evaluación de la eficacia, se compararon las medias de larvas de tercero y cuarto estadio 
de cada especie y las pupas (sin distinguir la especie) en cada muestreo para identificar las diferencias significativas entre las intervenciones y el testigo, y el efecto residual entre la época de alta y de baja pluviosidad. Se compararon con el promedio general y por fecha de muestreo quincenal. En el estudio se asumió que no existían diferencias entre las observaciones realizadas en los sumideros durante una misma semana debido a los cambios de precipitación.

Todos los datos se analizaron por medio de la regresión binomial negativa. Se utilizó esta prueba estadística debido a que la variable dependiente (número de larvas y pupas) corresponde a un conteo que presentó una dispersión amplia en la distribución de los datos (varianza mayor que el promedio). La variable independiente en este modelo correspondió a la intervención evaluada (triflumurón, VBC60035 y testigo). Un valor de $p<0,05$ se consideró como estadísticamente significativo. Se eliminaron de los análisis las observaciones fecha/sumidero que presentaron aceite, ya que esto elimina la presencia de mosquitos.

Además, para cada intervención se calculó: 1) la emergencia de las pupas en el laboratorio (sin diferenciar especie en pupa); 2) la relación de larvas y adultos de $A$. aegypti Vs. C. quinquefasciatus, y 3) el coeficiente de correlación de Spearman entre la cantidad de agua en un sumidero y la cantidad de estadios inmaduros evaluados.

\section{Resultados}

\section{Evaluación de la eficacia}

EI VBC 60035 fue el tratamiento más eficaz para el control de todos los estadios inmaduros, tanto de $A$. aegypti como de $C$. quinquefasciatus, y presentó diferencias muy significativas contra el testigo $(p<0,001)$. En contraste, el tratamiento con triflumurón sólo presentó diferencias significativas contra $A$. aegypti $(p<0,001)$ (cuadro 1).

El promedio de larvas recolectadas de $A$. aegypti, $C$. quinquefasciatus y pupas de ambas especies durante el seguimiento de las intervenciones, se observa en la figura 1. Los análisis por fecha de muestreo indican que, durante la época lluviosa (mayo 19 a junio 16, primeros 30 días), las poblaciones de larvas y pupas se mantienen bajas tanto en los tratamientos como el testigo. Durante esta época no se observaron diferencias significativas en la mayoría de las fechas de muestreos entre

Cuadro 1. Efecto de la aplicación quincenal de triflumuron y el VBC 60035 en A. aegypti y C. quinquefasciatus.

\begin{tabular}{|c|c|c|c|c|c|}
\hline & $\begin{array}{c}\text { A } \\
\text { Testigo }\end{array}$ & $\begin{array}{c}\text { B } \\
\text { Triflumurón }\end{array}$ & $\begin{array}{c}C \\
\text { VBC } \\
60035\end{array}$ & $\mathbf{z}$ & $\mathbf{p}^{*}$ \\
\hline Larvas de $A$. aegypti & $\begin{array}{c}21,31 \\
( \pm 44,37)\end{array}$ & $\begin{array}{c}4,53 \\
( \pm 14,54)\end{array}$ & $\begin{array}{c}5,78 \\
( \pm 7,57)\end{array}$ & $\begin{array}{l}-3,908 \\
-3,021 \\
0,792\end{array}$ & $\begin{array}{c}\text { A Vs. B } \\
<0,001 \\
\text { A Vs. C } \\
0,003 \\
\text { B Vs. C } \\
0,428\end{array}$ \\
\hline Larvas de $C$. quinquefasciatus & $\begin{array}{c}192,39 \\
( \pm 608,63)\end{array}$ & $\begin{array}{c}140,90 \\
( \pm 305,74)\end{array}$ & $\begin{array}{c}3,21 \\
( \pm 13,48)\end{array}$ & $\begin{array}{l}-0,140 \\
-7,700 \\
-9,120\end{array}$ & $\begin{array}{c}\text { A Vs. B } \\
0,889 \\
\text { A Vs. C } \\
<0,001 \\
\text { B Vs. C } \\
<0,001\end{array}$ \\
\hline Pupas de ambas especies & $\begin{array}{c}32,60 \\
( \pm 89,01)\end{array}$ & $\begin{array}{c}29,11 \\
( \pm 73,04)\end{array}$ & $\begin{array}{c}1,68 \\
( \pm 3,49)\end{array}$ & $\begin{array}{l}-0,053 \\
-6,283 \\
-6,202\end{array}$ & $\begin{array}{c}\text { A Vs. B } \\
0,957 \\
\text { A Vs. C } \\
<0,001 \\
\text { B Vs. C } \\
<0,001\end{array}$ \\
\hline
\end{tabular}

\footnotetext{
* Regresión binomial negativa, diferencias estadísticamente significativas con $p \leq 0,05$.
} 

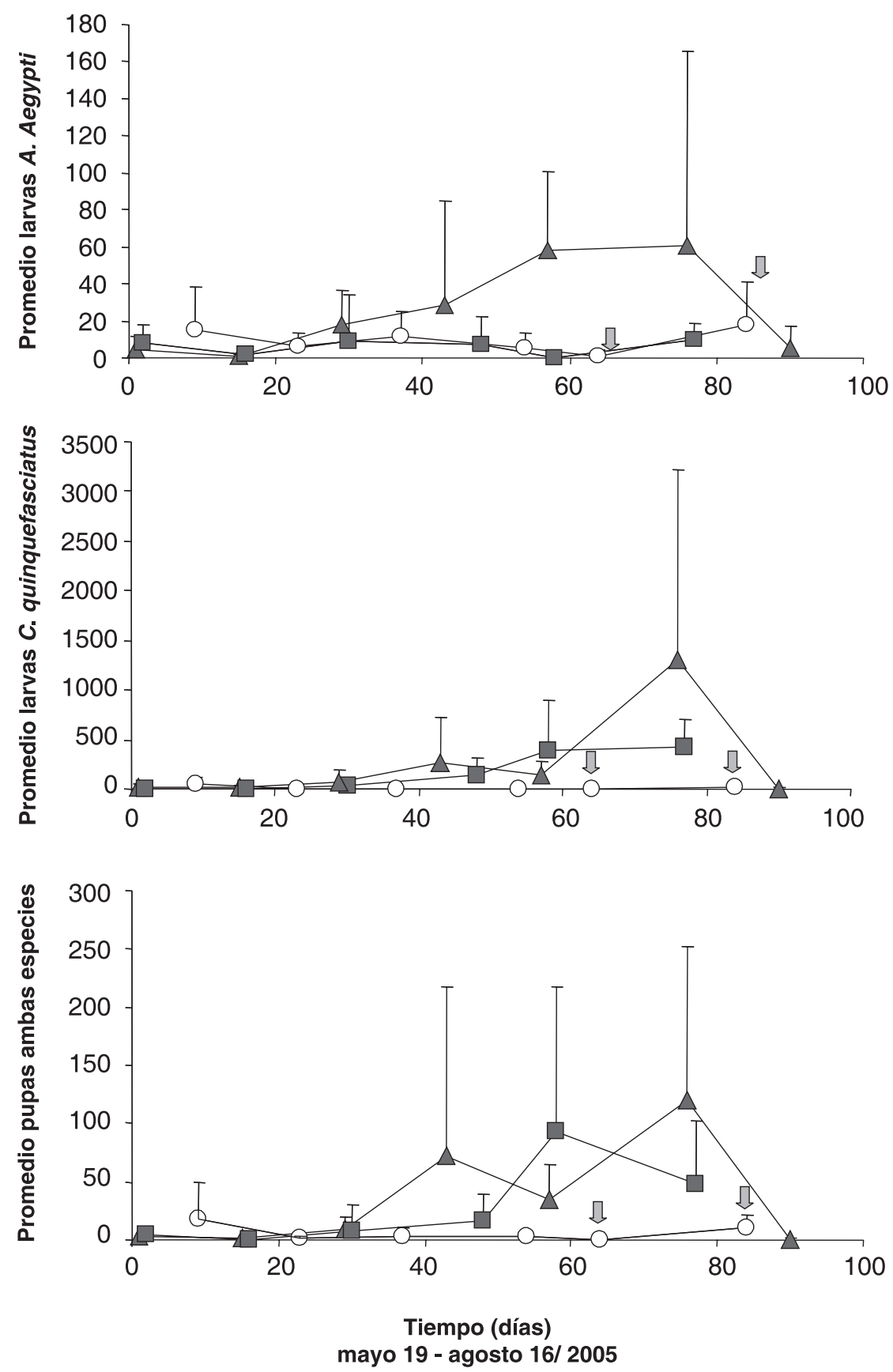

Testigo $\triangle \quad$ Triflumurón $\_$VBC 60035 - -

Figura 1. Dinámica de los estadios inmaduros durante aplicaciones quincenales del triflumurón y el VBC 60035, comparados con el testigo. Las barras verticales indican la desviación estándar. En cada visita quincenal se realizaba el muestreo y la aplicación de los larvicidas, excepto para las dos últimas fechas (indicado por las flechas), en las cuales no se aplicó el VBC 60035. 
las intervenciones y el testigo (no se presentan los datos).

Por el contrario, durante la época de baja pluviosidad se observaron diferentes comportamientos. El VBC 60035 presentó en todos los muestreos diferencias significativas en ambas especies en comparación con el triflumurón $(z=9,120, p<0,001)$ $y$ el testigo $(z=-7,700, p<0,001)$; mientras que el triflumurón sólo presentó diferencias contra el testigo en $A$. aegypti $(z=3,908, p<0,001)$ (no se presentan los datos). En todas las aplicaciones del VBC 60035 se observó $100 \%$ de mortalidad a las 72 horas después de la aplicación.

La relación de larvas de Culex/Aedes fue de 9,0 en el testigo, 31,1 en el triflumurón y 0,6 en el VBC 60035. Estas relaciones variaron al tener en cuenta la emergencia de adultos, en la cual el tratamiento con triflumurón presentó el mayor cambio de 31,1 a 349,5; mientras para el VBC 60035 y el testigo fue de 0,1 y 2,9 respectivamente. Lo anterior indica que la emergencia de $C$. quinquefasciatus fue muy alta con el tratamiento con triflumurón en comparación con Aedessp. La emergencia para pupas de ambas especies en los sumideros testigo y el VBC 60035 fue mayor del $85 \%$, mientras el promedio de emergencia para el triflumurón fue de $63 \%$.

\section{Efecto residual del VBC 60035}

El efecto residual del VBC 60035 sobre la dinámica de recolonización de $A$. aegypti y $C$. quinquefasciatus durante la época de alta y baja pluviosidad se observa en figura 2A. Al comparar el tratamiento entre las dos épocas, sólo se observaron diferencias estadísticamente significativas entre el promedio de larvas de $A$. aegypti $(z=5,279, p<0,001)$ (no se presentan los datos).

Al comparar el tratamiento de cada época con su correspondiente período del testigo, se observó que las mayores diferencias significativas se presentaron durante la época de baja pluviosidad en todos los estadios inmaduros ( $A$. aegypti: $z=14,577, p<0,001, C$. quinquefasciatus: $z=11,007$, $p<0,001$, pupas: $z=11,062, p<0,001$ ) (no se presentan los datos).

Durante la época de lluvias sólo se observaron diferencias estadísticamente significativas contra el testigo en larvas de $A$. aegypti $(\mathrm{z}=-3,19$, $p<0,001)$. Las diferencias observadas en la época de baja pluviosidad contra el testigo se mantuvieron durante todas las fechas de muestreo; sin embargo, se observa recolonización por $A$. aegypti y $C$. quinquefasciatus a partir de los 11 días después de la aplicación (figura 2A). La emergencia de las pupas recolectadas en ambas épocas fue similar a la zona testigo $(>85 \%)$.

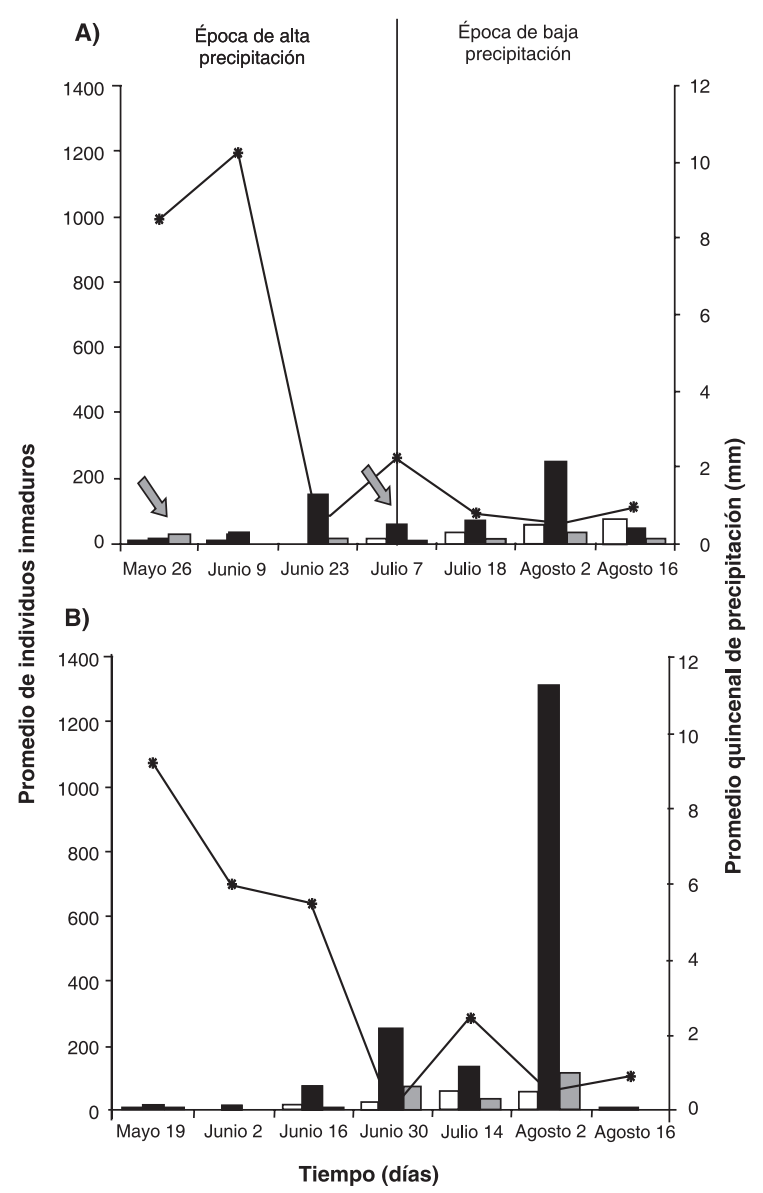

$\square$ Larvas A. aegypti Larvas C. quinquefasciatus

$\square$ Pupas de ambas especies $\rightarrow-$ Precipitación

Figura 2. A) Efecto residual de una única aplicación del VCB 60035. Fecha de aplicación indicada por las flechas. Los sumideros muestreados en la época de alta precipitación son diferentes a los de baja precipitación. B) Sumideros testigo. 


\section{Comportamiento de los sumideros testigo}

La dinámica de los diferentes estadios inmaduros en el testigo se observa en la figura 2B. Durante la época de alta pluviosidad los sumideros testigo presentaron bajo número de larvas y pupas, y se observó que en su mayoría no presentaron diferencias estadísticamente significativas con las intervenciones $(p>0,05)$. Mientras en la época de baja pluviosidad, en la mayoría de las revisiones, además de presentar diferencias muy significativas con las intervenciones $(p<0,001)$, el número de estadios inmaduros aumentó en forma directamente proporcional en el tiempo hasta llegar a un punto de saturación que produjo una caída de la población. La disminución en el promedio de larvas y pupas quizá se deba a que se ha llegado a la capacidad de carga $(K)$ del sumidero, es decir a que se ha alcanzado el número de individuos máximo que el ambiente puede sostener durante un período determinado (3).

En promedio, todos los sumideros del estudio presentaron el $61,37 \%$ de su capacidad total de agua y sólo uno se secó totalmente (no se presentan los datos). En ninguno de los tratamientos y para ninguno de los estadios inmaduros evaluados se encontró una correlación estadísticamente significativa entre el volumen de agua en los sumideros y el número de larvas y pupas recolectadas $(p>0,05)$.

\section{Discusión}

El tratamiento más eficaz para el control de los estadios inmaduros de mosquitos en los sumideros fue el VBC 60035, que controló ambas especies. El tratamiento actual con triflumurón sigue siendo efectivo contra larvas de $A$. aegyptipero no contra C. quinquefasciatus, como era sospechado por el organismo de control del dengue de la Secretaría de Salud Pública Municipal de Cali.

Las diferencias en la relación Culex/Aedes y el alto porcentaje en la emergencia de adultos de $C$. quinquefasciatus en el tratamiento con triflumurón $(63 \%)$, sugieren una gran resistencia de esta especie, ya que se espera que este tratamiento inhiba la emergencia de adultos debido a su efecto como inhibidor del crecimiento. Los resultados observados en el tratamiento con triflumurón contrastan con estudios anteriores realizados en Cali, en los que se encontró que este larvicida era efectivo contra $C$. quinquefasciatus (19) y $A$. aegypti $(19,22)$.

Esto indica que la aparición de la resistencia es reciente en las poblaciones de $C$. quinquefasciatus de Cali. La emergencia de la resistencia a este insecticida pudo deberse a la fuerte presión de selección por aplicaciones quincenales, el uso de este producto por más de seis años con cortas rotaciones con otros insecticidas (tres a seis meses) y la falta de evaluación periódica de las susceptibilidad a $C$. quinquefasciatus debido a que no era prioridad del programa de control de dengue.

Uno de los factores limitantes del diseño del estudio está relacionado con la selección de los sumideros a partir de zonas dentro del barrio y no a una selección aleatoria de intervención por cada sumidero; sin embargo, esto tenía factores limitantes logísticos. Dentro del estudio se asumió que el barrio era uniforme y que cada sumidero era una unidad independiente. Otra limitación del estudio fue el no haber identificado las pupas encontradas en los sumideros, determinación importante para futuros estudios.

La dinámica de las densidades de población de los estadios inmaduros de $A$. aegypti y $C$. quinquefasciatus en los sumideros varió con la estación climática en este estudio. En los sumideros testigo se observa que en la época lluviosa, el promedio de larvas y pupas se mantienen muy bajos en comparación con la época de baja pluviosidad, posiblemente por el recambio de agua (lavado y desbordamiento) producto de las lluvias constantes (figura 2B). Este comportamiento hizo que no existieran mayores diferencias entre los tratamientos y el testigo durante la época lluviosa. Esta evidencia sugiere que las actividades de control de los sumideros deben ser mayores en la época de baja pluviosidad, lo que favorecería el costo-efectividad de la estrategia.

El efecto residual del VBC 60035 varió de acuerdo con la estación climática evaluada, ya que durante el período de alta pluviosidad no se observaron diferencias significativas con el testigo a excepción de las larvas de $A$. aegypti. Mientras que durante la época de baja pluviosidad, se observaron 
diferencias muy significativas durante todo el período de evaluación en todos los estadios inmaduros evaluados. La evaluación del efecto residual durante la época de baja pluviosidad evidenció que la recolonización de $A$. aegypti y C. quinquefasciatus ocurrió a los 11 días después del tratamiento, ya que se observó la presencia de larvas y pupas (figura $2 \mathrm{~A}$ ).

Debido a que la recolonización de ambas especies ocurre tempranamente, se sugiere aplicar quincenalmente este producto para su control. Es posible que el efecto residual de la mezcla bacteriana en estos sumideros sea más corto que el sugerido por la casa comercial (un mes) debido a la alta contaminación de material químico (aceites) y orgánico (roedores muertos, excrementos de animales, hojas de árboles, basura plástica) observada.

Estos resultados difieren de los obtenidos por Suárez y Morales en 1998, quienes evaluaron la mezcla de Vectolex $\AA$ y Vectobac $\AA$ con $2 \mathrm{~g}$ de cada producto por sumidero, lo que produjo una reducción en pupas del $98 \%$ y $95 \%$ para Culex spp. y $A$. aegypti, respectivamente, durante 36 días en condiciones de absoluto verano (Suárez MF, Morales CA. Impacto de Bacillus sphaericus (Vectolex®) en poblaciones de Anopheles albimanus y Culex spp. en el Valle del Cauca. XXV Congreso de la Sociedad Colombiana de Entomología. Cali, Colombia, julio, 1998).

Las diferencias en el efecto residual pueden deberse a diferencias en las formulaciones, cantidad de ingrediente activo, diferencias en la sensibilidad al producto de las poblaciones evaluadas, o a que las diferentes áreas o barrios de la ciudad poseen diferentes niveles de infestación y contaminación; sin embargo, este trabajo no permite responder estas preguntas. Futuros estudios podrían estar enfocados a la aplicación de diferentes concentraciones y proporciones de la mezcla de $B$. thuringiensis var. israelensis y $B$. sphaericus para aumentar su efecto residual.

Los resultados de este estudio permiten confirmar que los sumideros de la ciudad de Cali son un criadero muy productivo de $C$. quinquefasciatus y $A$. aegypti, como fue descrito previamente por González y Suárez (González R, Suarez MF. Sewers: The principal Aedes aegypti breeding sites in Cali, Colombia. American Society of Tropical Medicine and Hygiene (ASTMH) Annual Meeting 44th. Texas, USA, November 17-21, 1995:217).

Se necesita la evaluación de diferentes alternativas químicas, orgánicas o cambios estructurales del sumidero para mejorar el control de estos mosquitos de importancia en salud pública en Cali. La búsqueda de insecticidas alternativos es necesaria para permitir la rotación de los mismos y disminuir la generación de resistencia, como fue observado con el producto triflumurón a $C$. quinquefasciatus. Se sugiere una vigilancia continua de la susceptibilidad a los insecticidas usados en estos sumideros para mantener estrategias de control que sean efectivas.

Los resultados del presente estudio fueron la base para implementar nuevas estrategias en el control de los sumideros Secretaría de Salud Pública Municipal de Cali, donde se comenzó a utilizar la mezcla de triflumurón con $B$. sphaericus, teniendo buenos resultados para el control de ambas especies. El VBC60035 podría ser empleado tanto por los organismos de control como en los programas de participación comunitaria debido al fácil manejo de la formulación (bolsas hidrosolubles).

\section{Agradecimientos}

Agradecemos la asesoría de Charles Apperson (North Carolina State University), Dawn Wesson (Tulane University), Ranulfo González (Universidad del Valle) y Peter DeChant (Valent BioSciences Corporation) en el diseño experimental de la propuesta. A Gilberto Londoño, por su acompañamiento y asistencia de campo y a los integrantes de la Unidad de Entomología Médica de CIDEIM, por su colaboración. A la Unidad de Epidemiología y Estadística de CIDEIM, por la asesoría en la creación de la base de datos y el análisis estadístico y a Beatriz Herrera de CIDEIM, por la consecución del material bibliográfico.

\section{Conflicto de intereses}

El estudio fue financiado por Valent BioSciences Corporation. Sin embargo, los investigadores tuvieron total independencia para el manejo y el análisis de los datos. 


\section{Financiación}

Este proyecto fue financiado por Valent BioSciences Corporation.

\section{Referencias}

1. Nelson MJ. Aedes aegypti: Biology and ecology. Washigton, D.C.: PAHO; 1986.

2. World Health Organization. Integrated vector control. Seventh Expert Committee on Vector Biology and Control. Technical Report Series 688. Geneva: WHO; 1983.

3. Woodring J, Davidson EW. Biological control of mosquitoes. En: Beaty BJ, Marquardt WC, editors. The biology of disease vectors. Colorado: University Press of Colorado; 1996. p. 530-48.

4. Wirth MC, Georghiou GP, Federici BA. CytA enables CrylV endotoxins of Bacillus thuringiensis to overcome high levels of CrylV resistance in the mosquito, Culex quinquefasciatus. Proc Natl Acad Sci USA. 1997;94:10536-40.

5. Cupp EW, Travi BL, González R. Filariasis. En: Travi BL, Montoya-Lerma J, editores. Manual de entomología médica para investigadores de América Latina. Cali: Centro Internacional de Entrenamiento e Investigaciones Médicas CIDEIM; 1994.

6. Fonseca DM, Keyghobadi N, Malcolm CA, Mehmet C, Schaffner F, Mogi M, et al. Emerging vectors in the Culex pipiens complex. Science. 2004;303:1535-8.

7. Suárez-Rubio $\mathbf{M}$, Suárez $\mathbf{M}$. The use of the copepod Mesocyclops longisetus as a biological control agent for Aedes aegypti in Cali, Colombia. J Am Mosq Control Assoc. 2004;20:401-4.

8. De la Fuente J. Zoología de los artópodos Madrid: McGraw-Hill Interamericana; 1994.

9. Batra CP, Mittal PK, Adak T, Ansari MA. Efficacy of IGR compound Starycide 480 SC (Triflumuron) against mosquito larvae in clear and polluted water. $\mathrm{J}$ Vector Borne Dis. 2005;42:109-16.

10. Darriet F, Carnevale P, Robert V. Laboratory and field evaluation of the activity of an ecdysoid-type insect growth inhibitor, Triflumuron (OMS-2015), on Culex quinquefasciatus, Anopheles gambiae and Aedes aegypti. Geneva: World Health Organization; 1985.

11. Regis L, Silva-Filha MH, Nielsen-LeRoux C, Charles JF. Bacteriological larvicides of dipteran disease vectors. Trends Parasitol. 2001;17:377-80.
12. Instituto Geográfico Agustín Codazzi. Diccionario geográfico de Colombia. Segunda edición. Tomo I. Bogotá: Subdirección de Investigación y Divulgación Geográfica; 1980.

13. Ministerio de la Protección Social. SIVIGILA, Sistema Nacional de Vigilancia en Salud Pública. Prevención y control del dengue. Informe Ejecutivo Semanal. 2001 Semana epidemiológica No. 42. Octubre 14 al 20 de 2001.

14. Ministerio de la Protección Social. SIVIGILA, Sistema Nacional de Vigilancia en Salud Pública. Comportamiento por regiones del dengue en el 2001. 2002 Semana Epidemiológica No. 2. Enero 06 a 12 de 2002.

15. Laguado J, Alvis $\mathbf{N}$, Máttar $\mathbf{S}$. Investigación de un brote de fiebre de origen desconocido en una localidad colombiana del Caribe. Colombia Médica 2005;36:25462.

16. IDEAM-Estación Base Aérea Marco Fidel Suárez. Valores diarios de precipitación, humedad relativa y temperatura. Bogotá, D.C.: Instituto de Hidrología, Meteorología y Estudios Ambientales (IDEAM); 2005.

17. Sih A. Antipredator responses and the perception of danger by mosquito larvae. Ecology. 1986;67:434-41.

18. Satizábal Rengifo ME. Control biológico de Aedes aegypti (Diptera: Culicidae) con Mesocyclops longisetus (Copepoda: Cyclopoida) en sumideros de la ciudad de Cali, Colombia (tesis). Cali: Universidad del Valle; 1999.

19. Morales CA. Evaluación de la eficacia de triflumurón (ingrediente activo de Starycide ${ }^{\circledR}$ ) en el control de larvas de Aedes aegypti y Culex spp. en sumideros de Cali, Colombia. Salud Pública-Bayer. 2002;17:66-71.

20. Centers for Disease Control and Prevention. Pictorial keys to arthropods, reptiles, birds and mammals of public health significance. U.S. Departament of health \& human services-CDC. Disponible en: http://www.cdc.gov/nceh/ehs/ Pictorial_Keys.htm

21. Lane R. Neotropical Culicidae. Dixinae, Chaoborinae and Culicinae, tribes Anophelini, Toxorhynchitini and Culicini (Genus Culex only). Sao Paulo: University of Sao Paulo; 1953.

22. Prieto AV, Suárez MF, González R. Suceptibilidad de dos poblaciones de Aedes aegypti (Diptera:Culicidae) de Cali (Valle, Colombia) a Temefos (Abate $\left.{ }^{\circledR}\right)$ y Triflumuron (Starycide ${ }^{\circledR}$ ). Revista Colombiana de Entomología. 2002;28:175-8. 